\title{
Functional End Groups in Living Ring-Opening Metathesis Polymerization
}

\author{
Andreas F. M. Kilbinger*(iD) \\ University of Fribourg, Chemistry Department, Chemin du Musée 9 \\ 1700 Fribourg, Switzerland \\ andreas.kilbinger@unifr.ch
}

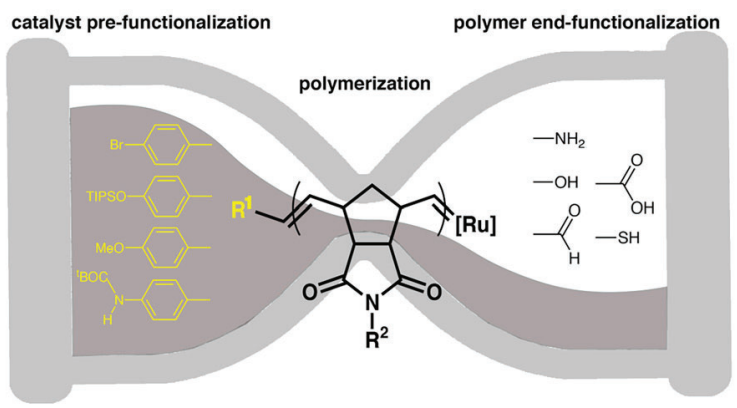

Abstract Over the last two decades many synthetic methods have been reported to selectively introduce a number of different functional groups at the chain end of a living ring-opening metathesis polymer. In this personal account, I would like to focus on a few such methods developed in my research group over the last several years and how these led to the discovery of catalytic living ring-opening metathesis polymerization, a ring-opening metathesis polymerization method controlled by the polymer end groups. This account consists of the following sections:

Introduction

Functionalization of the Propagating Chain End

Functionalization of the Initiating Chain End

Polymerization Control by Functional End Groups

Conclusions

Keywords Grubbs catalyst, ring-opening metathesis, polymer end groups, degenerative chain transfer, polymerization

\section{Introduction}

For the purpose of this account I would like to define a mono-telechelic polymer as one which carries exactly one reactive functional group at one polymer chain end which is different from all other functional groups that might be present in the polymer chain. In a homo-telechelic polymer two identical reactive functional groups are present at either chain end and in a hetero-telechelic polymer, the two functional groups at either chain end are different, i.e. can be addressed individually. To distinguish between the two chain ends of a polymer I will use the terms 'initiating chain end' for the one that started the chain and 'propagating chain end' for the one through which chain growth is achieved.
Polymers in which one or both chain ends can be used in subsequent reactions have attracted significant interest since the term 'telechelic' was first introduced to polymer chemistry in $1960 .{ }^{1}$ Such polymers can, for example, be used as macromonomers in subsequent step-growth processes such as polyurethane syntheses. They can form the starting materials to prepare more complex polymeric architectures such as block, star, or graft-copolymers. ${ }^{2}$ Homo telechelic polymers have also been extensively used in the fields of metal-coordinated polymers ${ }^{3}$ and supramolecular polymers. ${ }^{4}$ Furthermore, monotelechelic polymers are used to graft onto macroscopic or nanoparticle surfaces.

Many modern living polymerization reactions ${ }^{5}$ yield end-functional polymer chains. In living carbanionic polymerization the terminal propagating carbanion can be exploited to yield many different functional end groups by reaction with suitable electrophiles. ${ }^{6}$ Living carbocationic polymerization can, similarly, be employed for the introduction of a variety of functional chain ends. ${ }^{7}$ Controlled radical polymerizations are highly popular today as they can be carried out easily while avoiding the rigorously purified reagents and solvents that are typically required for ionic polymerizations. ${ }^{8}$ Atom transfer radical polymerization (ATRP) polymers mostly carry an aliphatic halide end group which can undergo nucleophilic substitution for derivatization. ${ }^{9}$ Reversible addition fragmentation chain transfer (RAFT) polymers ${ }^{10}$ that carry dithioester or thiocarbonate end groups can equally easily be converted into a number of different functional chain end groups. ${ }^{11}$ Living ring-opening polymerizations of heterocycles such as epoxides, lactones, lactams, and others can all yield heteroatomcontaining end groups, for example alcohols, amines, and many others.

In my group, we have been interested in finding new ways of introducing reactive functional groups at the chain ends of polymers prepared by living ring-opening metathe- 
sis polymerization (ROMP) using Grubbs-type ruthenium carbene complexes (Scheme 1, top). ${ }^{12}$ Methods to end-functionalize metathesis polymers by using other metal carbenes are not discussed in this account. ${ }^{13}$ When we entered this field in 2006, there were only a few reported methods available for the chain end-functionalization of living ROMP polymers. These approaches were mainly based on enol ether derivatives that react with a propagating ruthenium carbene chain end to transfer a functional group to the polymer chain end with concomitant formation of a Fischer carbene complex (Scheme 2) ${ }^{14}$ As the Fischer carbene complex shows significantly reduced olefin metathesis activity compared to the propagating alkylidene species this can be seen as an example of a functional termination reaction.

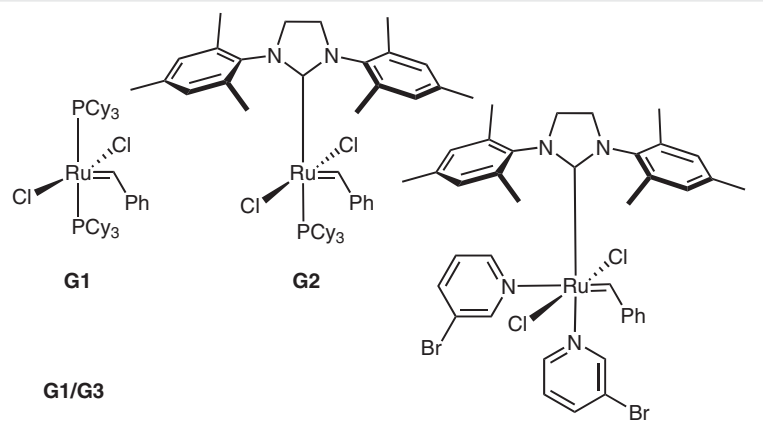

G3

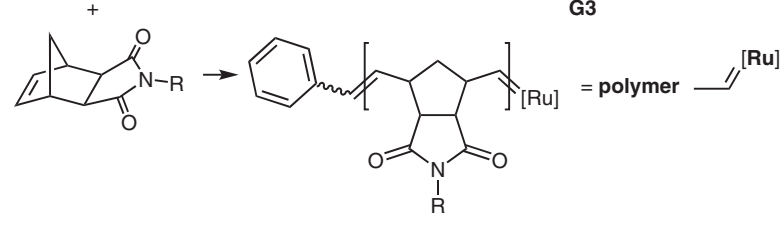

$\mathrm{R}=$ methyl, phenyl, hexyl, 2-ethylhexyl

Scheme 1 Top: Grubbs $1^{\text {st }}$ generation (G1), second generation (G2) and third generation (G3) olefin metathesis complexes. Bottom: A typical living ring-opening polymerization initiated with $\mathrm{G} 1$ or $\mathrm{G} 3$.

The groups of Grubbs, Kiessling, and others had used this method extensively to introduce functional groups such as azides, fluorophores, chromophores, or other (bio)active groups to the chain ends of living ROMP polymers. ${ }^{15}$

Within this account, the term 'living polymer' refers to a living ROMP polymer such as the one shown in Scheme 1 (bottom). Monomers such as norbornene-5,6-dicarboximides typically propagate in a living manner. There, the ringopened backbone olefins show virtually no tendency to undergo further olefin metathesis reactions because of steric crowding. The absence of secondary metathesis reactions constitutes a fundamental requirement to observe living behavior in a ring-opening metathesis polymerization.

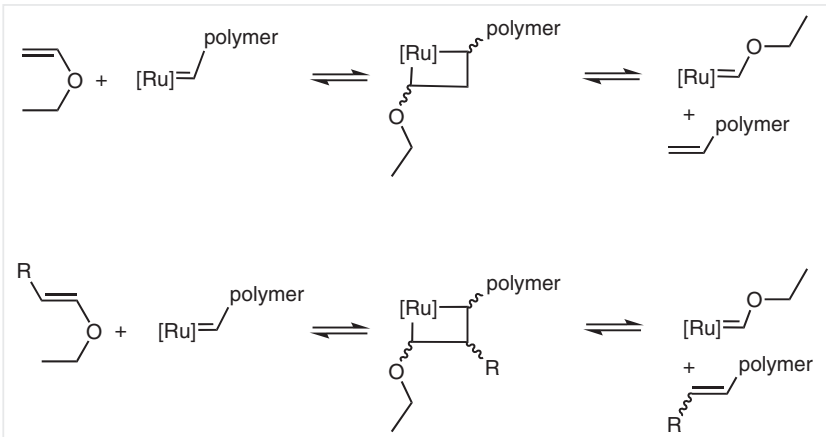

Scheme 2 Nonfunctional and functional termination of Ru-based olefin metathesis polymerizations with use of enol ether derivatives

\section{Functionalization of the Propagating Chain End}

Our first attempt to functionalize living ROMP polymers also focused on the propagating chain end of a polymer. This required the conversion of the ruthenium carbene complex into a new functional group after consumption of all monomer. We used a commercial Grubbs $1^{\text {st }}$ generation ruthenium benzylidene complex as initiator to polymerize exo- $N$-phenyl-norbornene-5,6-dicarboximide followed by polymerization of a dioxepine derivative (Scheme 3). ${ }^{16} \mathrm{Re}-$ moval of the ruthenium complex from the propagating polymer chain end by using ethyl vinyl ether yielded a di- 
block copolymer which, after acid hydrolysis of the acetal groups, gave a mono-end-functional ROMP polymer with exactly one terminal allylic alcohol group.

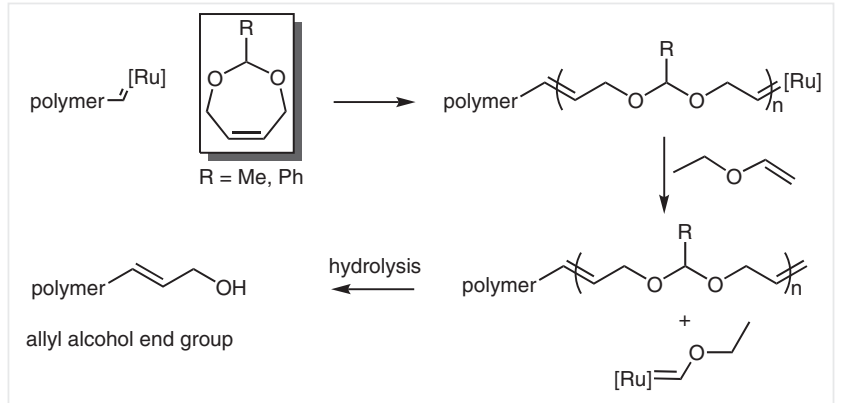

alternative reagents:
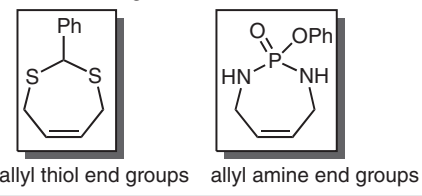

allyl thiol end groups allyl amine end groups

Scheme 3 Sacrificial block copolymer synthesis. After hydrolysis, alcohol, thiol, or amine end groups are obtained.

An advantage of this method is that the successful formation of the precursor diblock copolymer as well as the final mono telechelic polymer can be easily followed by size exclusion chromatography (SEC). A considerable disadvantage is of course the poor atom economy of this synthetic strategy. ${ }^{17}$ We could also show that terminal allylic thiols ${ }^{18}$ and allylic amines ${ }^{19}$ were accessible using similar reaction pathways (Scheme 3, bottom). Recently, the group of Xia showed that functionally substituted 1,1-cyclopropenes undergo a single addition to a propagating polymer chain. ${ }^{20}$ This allows an atom-economic chain-end-functionalization and opens up the possibility to further exploit the reactivity of the propagating chain end.

A real functional termination without the intermediate formation of a diblock copolymer was achieved by using a cyclic enol ester and vinylene carbonate (Scheme 4A, 4B). ${ }^{21}$ Similar to enol ethers, enol esters form Fischer carbenes by reacting with propagating ruthenium alkylidene complexes. While these Fischer carbenes show a slightly higher olefin metathesis activity than those based on enol ethers, they can still be considered metathesis inactive under typical polymerization conditions under which the enol ester is added at the end of the polymerization in large excess.

As shown in Scheme $4 \mathrm{~A}$ and $4 \mathrm{~B}$ the propagating ruthenium carbene complex undergoes ring-opening of these cyclic enol esters, thereby forming a deactivated Fischer carbene complex. Fischer carbene-Ru complexes from enol esters spontaneously form ruthenium-carbido complexes in dichloromethane, which results in monotelechelic polymer chains with either aldehyde or carboxylic acid end groups as depicted in Scheme 4. Both, G1- and G3-initiated polymers could be used for this polymer end-functionalization reaction.

In analogy to the cyclic enol esters described above, we also prepared acyclic enol ester derivatives for polymer end-functionalization (Scheme 4C). ${ }^{22}$ Such compounds can be readily prepared from a large range of commercially available functional aldehydes or alcohols and introduce the desired functionality to the monotelechelic polymer chain end similar to the enol ether derivatives mentioned above (Scheme 2).

A slightly different mechanistic approach was taken when reactions of propagating ruthenium alkylidenes (G1 or G3) were performed with 2-methoxy-3,4-dihydro-2Hpyran (MDHP, Scheme 5). Upon ring opening of this reagent

A

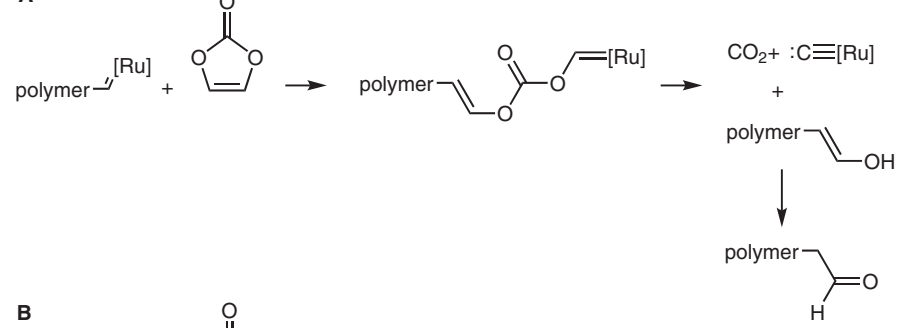

$\rightarrow$ polymer

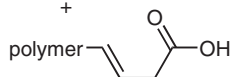

C

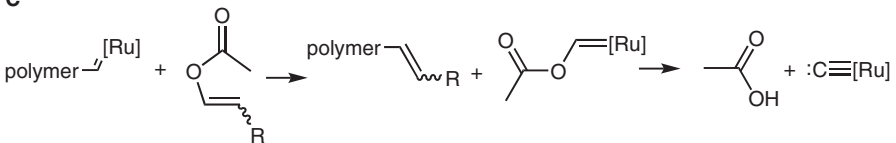

Scheme 4 Polymer end-functionalization with cyclic and acyclic enol esters 


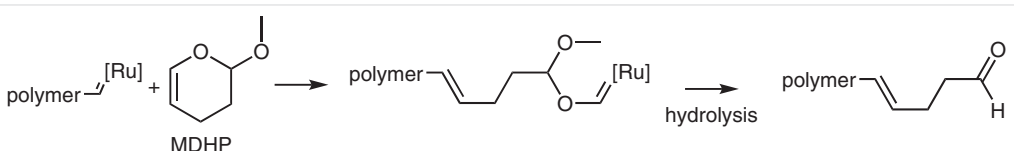

Scheme 5 Aldehyde end groups from reaction with cyclic enolacetals

a Fischer carbene complex is formed as an acetal which can be hydrolyzed under controlled conditions to form a monotelechelic polymer with a terminal aldehyde group. ${ }^{23}$ The commercial availability and low cost of this reagent make it particularly interesting for a ROMP end-functionalization method. Furthermore, the chemical versatility of aldehyde end groups can readily be exploited in reduction, oxidation reductive amination, and others to create new functional end groups or attach larger molecules to the polymer chain end.

\section{Functionalization of the Initiating Chain End}

All of the end-functionalization strategies mentioned so far focused on the chemical modification of the terminal chain end, i.e. the reaction of the propagating rutheniumcarbene complex after complete monomer consumption. The modification of the starting/initiating chain end would be even more useful as the polymer chain could be initiated with a functional initiator and subsequently terminated with a functional terminating agent yielding, in principle, two different functional groups at either chain end, i.e. a heterotelechelic polymer.

The metathesis-active carbene ligand of Grubbs $3^{\text {rd }}$ generation ruthenium benzylidene complex can easily be exchanged by reaction with excess of a symmetric acyclic olefin. This strategy was used by Matson et al. and us to prepare homotelechelic narrow polydispersity polymers. ${ }^{24}$ Heterotelechelic polymers are, unfortunately, not accessible by using this method because the excess symmetric olefin necessary for the initial cross-metathesis reaction to prefunctionalize the ruthenium carbene complex will also terminate the propagating chain end with the same functional group after all monomer has been consumed. As the propagation reaction of the highly ring-strained norbornene derivative is much faster than the cross-metathesis with the excess of acyclic symmetric olefin, good chain length control of the polymers can be achieved up to $10,000 \mathrm{~g} \mathrm{~mol}^{-1}$.

If the concentration of acyclic olefin is increased in such a polymerization reaction, the rate of cross-metathesis can be increased to such an extent that it occurs even before all strained monomers such as norbornene derivatives have been consumed. The chain length of the polymer is then controlled by the relative rates of propagation and crossmetathesis. Use of a symmetric acyclic olefin as cross-metathesis partner will give a homotelechelic polymer. For an unsymmetric olefin, a mixture of homo- and heterotelechelic polymers would be expected unless the cross-metathesis reaction occurs in a regioselective manner (Scheme 6).

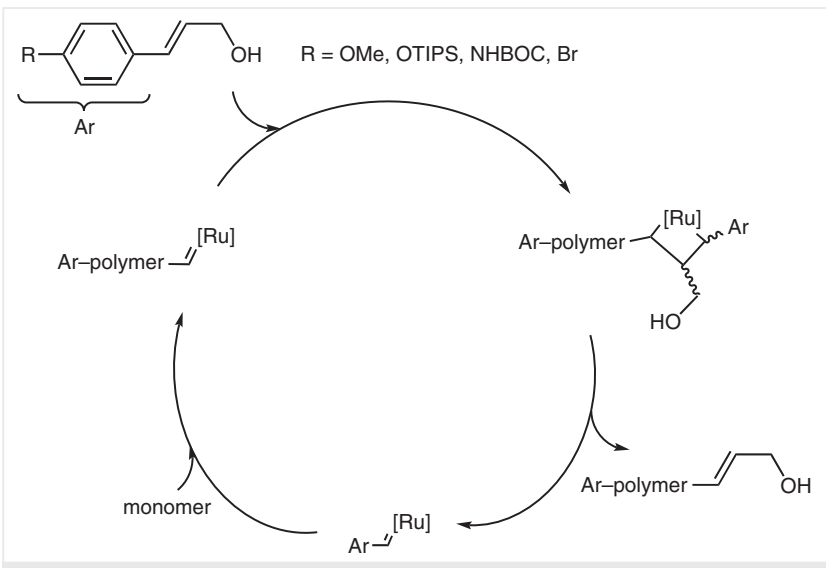

Scheme 6 Regioselective cross-metathesis for kinetically controlled heterotelechelic polymer synthesis

We recently found that cinnamic alcohol and derivatives thereof react in a regioselective manner with polymers initiated by Grubbs $1^{\text {st }}$ generation ruthenium complex G1. In these cases, the ruthenium benzylidene complex is reformed when a propagating polymer chain reacts with the cinnamic alcohol derivative. This means that the allylic alcohol part of cinnamic alcohol gets transferred to the polymer chain end while the newly formed ruthenium benzylidene complex will initiate the propagation of a new polymer chain. Using aryl-substituted cinnamic alcohol derivatives allowed us to prepare heterotelechelic polymers from norbornene derivatives under kinetic control. ${ }^{25}$

For a clean pre-functionalization of the commercial Grubbs benzylidene catalysts one would ideally require a reagent that, even when used in excess, would not interfere with the subsequent propagation or the final functional termination of the polymer.

Scheme 7A shows a 7-syn-allylether-substituted norbornene, a reagent that fulfills these criteria to a large extent. When reactions were performed with $\mathbf{G 1}$ or $\mathbf{G} 3$ benzylidene complexes the norbornene derivative is first ringopened, followed by a ring-closing metathesis towards the allylic ether double bond thereby yielding a new ruthenium-carbene complex. ${ }^{26}$ Any excess of the 7-syn-substituted norbornene derivative will be turned over by the newly formed ruthenium-carbene complex while regenerating itself. The hexahydrocyclopenta[b]pyran (HCP) derivative 
side product shows a much lower metathesis reactivity compared to norbornene derivatives. However, the propagating polymer chain end will react with HCP once all the strained and more reactive monomer has been consumed (Scheme 7). If 2-methoxy-3,4-dihydro-2H-pyran (MDHP) is added to the prefunctionalized ruthenium carbene initiator at the same time as the strained monomer is introduced, the polymer chain will be functionally initiated, consume all monomer through propagation and then react with MDHP which is less reactive than the monomer but significantly more reactive than HCP. In this way, a heterotelechelic polymer with narrow molecular weight dispersity could be synthesized.
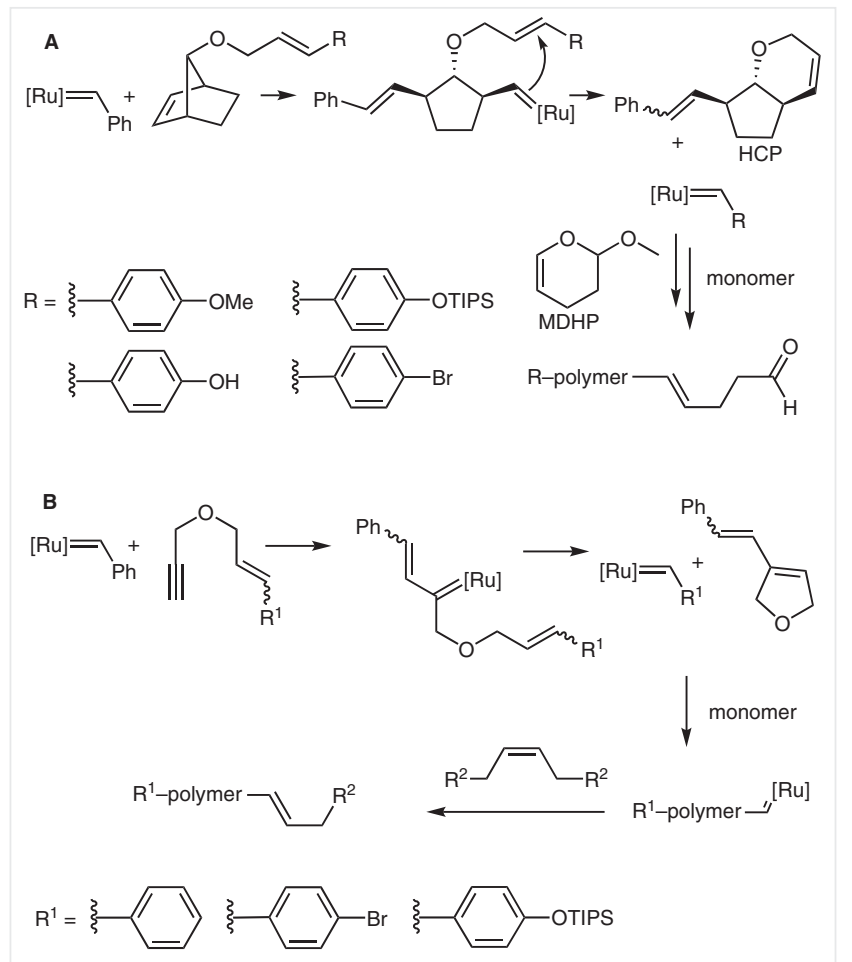

$\mathrm{R}^{2}=\mathrm{OH}, \mathrm{Cl}, \mathrm{NHBOC}$

Scheme 7 Heterotelechelic polymers through pre-functionalization of Ru complexes

An alternative approach using a synthetically more readily accessible pre-functionalization agent was recently reported by us $^{27}$ and shortly after by the Gutekunst group. ${ }^{28}$ Here, a propargylic-allyl-ether derivative is used to rapidly trap the commercial $\mathbf{G} 3$ benzylidene complex through a metathesis reaction with the alkyne (Scheme 7B). This quick first reaction with the alkyne has the advantage over the method described above (Scheme 7A) that the newly generated ruthenium-carbene complexes-which are potentially more reactive than the original benzylidene complex-can be formed in higher yield as they can no longer compete for the propargylic-allyl-ether pre-functionaliza- tion agent which has already been consumed. The 2,5-dihydrofuran side product formed from the original propargylic allyl ether did not interfere with propagation or functional termination reactions contrary to the HCP described above (Scheme 7A).

\section{Polymerization Control by Functional End Groups}

Upon closer investigation of the HCP side product discovered in the previous investigation, we realized that HCP can react with propagating ruthenium-alkylidene complexes in an equilibrium ring-opening-ring-closing sequence, thereby regenerating the exact same bicyclic structure and a ruthenium-benzylidene complex (Scheme 8). If propagating ruthenium-carbene complexes present on either side of this equilibrium could exchange rapidly with each other, the conditions of a degenerate chain transfer mechanism would be fulfilled. This is similar to the one commonly used in RAFT polymerizations.

As shown in Scheme 8, a ruthenium-carbene complex propagating polymer1 reacts with the HCP-like end group of polymer2 under ring opening to form the intermediate shown in Scheme 8. This ruthenium-carbene complex can either ring-close towards polymer 1 (Scheme 8, top equilibrium) or towards polymer2 (Scheme 8 , bottom equilibri$\mathrm{um}$ ), thereby essentially exchanging polymer end groups between polymer1 and polymer2. If this exchange of end groups is fast compared to the propagation reaction, a substoichiometric amount of ruthenium carbene complex is sufficient for the apparent propagation of many polymer chains at the same time.

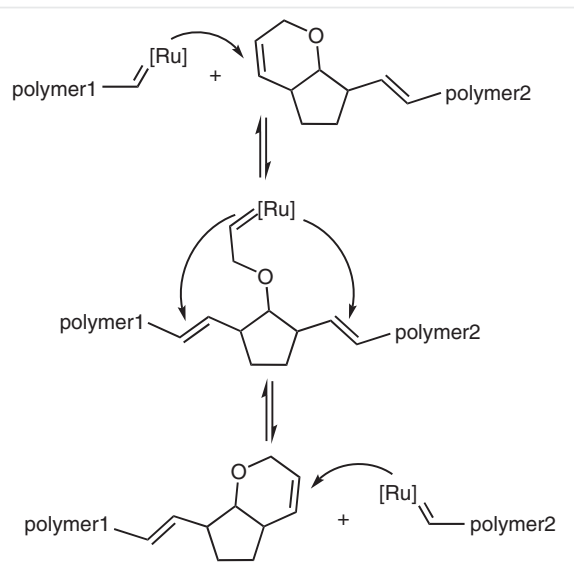

Scheme 8 Catalytic living ROMP, a degenerative chain transfer mechanism

The major problem in this case, however, is the relatively low reactivity of the HCP-like bicyclic chain transfer agent located at the chain end with respect to the reactivity of the highly strained monomer. For a degenerative chain transfer mechanism to be able to control the polymer mo- 
lecular weight, a rapid exchange of propagating chain ends must be ensured. In other words, the rate of propagation must not be higher than the rate of exchange of propagating chain ends. To achieve this goal, we simply added the monomer slowly to the reaction mixture with a syringe pump, thereby artificially lowering the rate of reaction. ${ }^{29}$

This new method of catalytic living ROMP is particularly useful if low molecular weight polymers are targeted. In a classical living ROMP, stoichiometric amounts of ruthenium-carbene complexes, such as G1 or G3, must be employed with respect to the number of polymer chains aimed for. For short polymer chains, this can lead to a rather high amount of ruthenium complex, which increases the cost and contaminates the final polymer as far as cell toxicity and color are concerned, for example. Using catalytic living ROMP, we have already managed to reduce the amount of ruthenium by a factor of 100 while maintaining good control over the molecular weight and molecular weight dispersity. Furthermore, the initiation kinetics of the individual metathesis complex do not appear to be important under catalytic living ROMP conditions. In addition to using the G3 complex, narrow polydispersity polymers can even be prepared by employing metathesis complexes such as G2 which, under noncatalytic conditions, would yield broad molecular weight dispersities with little control over the molecular weight.

\section{Conclusions}

This personal account of the work carried out by the author's research group in the area of end-group functionalization of living ring-opening metathesis polymers gives an overview over some of the synthetic methods that have been employed to achieve homo- and heterotelechelic ROMP polymers with varying degrees of molecular weight and/or dispersity control. Most recently, our research efforts in this area have allowed us to use functional polymer end groups to control the polymerization, i.e. the propagation reaction itself exploiting a reversible chain transfer mechanism. Current research efforts focus on the development of new chain transfer agents for catalytic living ROMP.

\section{Funding Information}

The author thanks the Swiss National Science Foundation and the Fribourg Centre for Nanomaterials (Frimat) for financial support.

\section{Acknowledgment}

The author thanks Mr. Mohammad Yasir and Mr. Subhajit Pal for helpful discussions regarding this manuscript.

\section{References}

(1) Uraneck, C. A.; Hsieh, H. L.; Buck, O. G. J. Polym. Sci. 1960, 46, 535.

(2) (a) Lo Verso, F.; Likos, C. N. Polymer 2008, 49, 1425. (b) Iha, R. K.; Wooley, K. L.; Nystrom, A. M.; Burke, D. J.; Kade, M. J.; Hawker, C. J. Chem. Rev. 2009, 109, 5620.

(3) (a) Winter, A.; Schubert, U. S. Chem. Soc. Rev. 2016, 45, 5311. (b) Li, W.; Kim, Y.; Li, J. F.; Lee, M. Soft Matter 2014, 10, 5231.

(4) (a) Folmer, B. J. B.; Sijbesma, R. P.; Versteegen, R. M.; van der Rijt, J. A. J.; Meijer, E. W. Adv. Mater. 2000, 12, 874. (b) Wei, M. H.; Li, B. Y.; David, R. L. A.; Jones, S. C.; Sarohia, V.; Schmitigal, J. A.; Kornfield, J. A. Science 2015, 350, 72. (c) de Espinosa, L. M.; Fiore, G. L.; Weder, C.; Foster, E. J.; Simon, Y. C. Prog. Polym. Sci. 2015, 49-50, 60. (d) Brunsveld, L.; Folmer, B. J. B.; Meijer, E. W.; Sijbesma, R. P. Chem. Rev. 2001, 101, 4071.

(5) Grubbs, R. B.; Grubbs, R. H. Macromolecules 2017, 50, 6979.

(6) (a) Jagur-Grodzinski, J. J. Polym. Sci., Part A: Polym. Chem. 2002, 40, 2116. (b) Yu, Y. G.; Chae, C. G.; Kim, M. J.; Seo, H. B.; Grubbs, R. H.; Lee, J. S. Macromolecules 2018, 51, 447. (c) Hirao, A.; Hayashi, M. Acta Polym. 1999, 50, 219.

(7) (a) Feldthusen, J.; Ivan, B.; Muller, A. H. E.; Kops, J. J. Macromol. Sci., Pure Appl. Chem. 1995, A32, 639. (b) Sawamoto, M.; Enoki, T.; Higashimura, T. Polym. Bull. (Berlin) 1987, 18, 117. (c) Aoshima, S.; Kanaoka, S. Chem. Rev. 2009, 109, 5245.

(8) (a) Pan, X. C.; Fantin, M.; Yuan, F.; Matyjaszewski, K. Chem. Soc. Rev. 2018, 47, 5457. (b) Chmielarz, P.; Fantin, M.; Park, S.; Isse, A. A.; Gennaro, A.; Magenau, A. J. D.; Sobkowiak, A.; Matyjaszewski, K. Prog. Polym. Sci. 2017, 69, 47. (c) Pan, X. C.; Tasdelen, M. A.; Laun, J.; Junkers, T.; Yagci, Y.; Matyjaszewski, K. Prog. Polym. Sci. 2016, 62, 73. (d) Tsarevsky, N. V.; Matyjaszewski, K. Chem. Rev. 2007, 107, 2270. (e) Pintauer, T.; Matyjaszewski, K. Chem. Soc. Rev. 2008, 37, 1087. (f) Tasdelen, M. A.; Kahveci, M. U.; Yagci, Y. Prog. Polym. Sci. 2011, 36, 455.

(9) (a) Anastasaki, A.; Willenbacher, J.; Fleischmann, C.; Gutekunst, W. R.; Hawker, C. J. Polym. Chem. 2017, 8, 689. (b) Siegwart, D. J.; Oh, J. K.; Gao, H. F.; Bencherif, S. A.; Perineau, F.; Bohaty, A. K.; Hollinger, J. O.; Matyjaszewski, K. Macromol. Chem. Phys. 2008, 209, 2180. (c) Gutekunst, W. R.; Anastasaki, A.; Lunn, D. J.; Truong, N. P.; Whitfield, R.; Jones, G. R.; Treat, N. J.; Abdilla, A.; Barton, B. E.; Clark, P. G.; Haddleton, D. M.; Davis, T. P.; Hawker, C. J. Macromol. Chem. Phys. 2017, 218, 1700107.

(10) Junkers, T. J. Polym. Sci., Part A: Polym. Chem. 2011, 49, 4154.

(11) (a) Willcock, H.; O'Reilly, R. K. Polym. Chem. 2010, 1, 149. (b) Moad, G.; Rizzardo, E.; Thang, S. H. Polym. Int. 2011, 60, 9. (c) Roth, P. J.; Haase, M.; Basche, T.; Theato, P.; Zentel, R. Macromolecules 2010, 43, 895. (d) Moad, G.; Chong, Y. K.; Postma, A.; Rizzardo, E.; Thang, S. H. Polymer 2005, 46, 8458.

(12) (a) Bielawski, C. W.; Grubbs, R. H. Angew. Chem. Int. Ed. 2000, 39, 2903. (b) Choi, T. L.; Grubbs, R. H. Angew. Chem. Int. Ed. 2003, 42, 1743. (c) Dias, E. L.; Nguyen, S. T.; Grubbs, R. H. J. Am. Chem. Soc. 1997, 119, 3887.

(13) Chen, Y. J.; Abdellatif, M. M.; Nomura, K. Tetrahedron 2018, 74, 619.

(14) Wu, Z.; Nguyen, S. T.; Grubbs, R. H.; Ziller, J. W. J. Am. Chem. Soc. 1995, 117, 5503.

(15) (a) Gordon, E. J.; Gestwicki, J. E.; Strong, L. E.; Kiessling, L. L. Chem. Biol. 2000, 7, 9. (b) Owen, R. M.; Gestwicki, J. E.; Young, T.; Kiessling, L. L. Org. Lett. 2002, 4, 2293. (c) Kolonko, E. M.; Kiessling, L. L. J. Am. Chem. Soc. 2008, 130, 5626. (d) Matson, J. B.; Grubbs, R. H. Macromolecules 2008, 41, 5626.

(16) Hilf, S.; Berger-Nicoletti, E.; Grubbs, R. H.; Kilbinger, A. F. M. Angew. Chem. Int. Ed. 2006, 45, 8045. 
(17) Hilf, S.; Grubbs, R. H.; Kilbinger, A. F. M. Macromolecules 2008, $41,6006$.

(18) Hilf, S.; Kilbinger, A. F. M. Macromolecules 2009, 42, 4127.

(19) Nagarkar, A. A.; Crochet, A.; Fromm, K. M.; Kilbinger, A. F. M. Macromolecules 2012, 45, 4447.

(20) Elling, B. R.; Xia, Y. ACS Macro Lett. 2018, 7, 656.

(21) Hilf, S.; Grubbs, R. H.; Kilbinger, A. F. M. J. Am. Chem. Soc. 2008, 130,11040

(22) Liu, P.; Yasir, M.; Kurzen, H.; Hanik, N.; Schafer, M.; Kilbinger, A. F. M. J. Polym. Sci., Part A: Polym. Chem. 2017, 55, 2983.

(23) Nagarkar, A. A.; Kilbinger, A. F. M. Chem. Sci. 2014, 5, 4687.
(24) (a) Matson, J. B.; Virgil, S. C.; Grubbs, R. H. J. Am. Chem. Soc. 2009, 131, 3355. (b) Hanik, N.; Kilbinger, A. F. M. J. Polym. Sci., Part A: Polym. Chem. 2013, 51, 4183.

(25) Liu, P.; Yasir, M.; Ruggi, A.; Kilbinger, A. F. M. Angew. Chem. Int. Ed. 2018, 57, 914.

(26) Nagarkar, A. A.; Yasir, M.; Crochet, A.; Fromm, K. M.; Kilbinger, A. F. M. Angew. Chem. Int. Ed. 2016, 55, 12343.

(27) Pal, S.; Lucarini, F.; Ruggi, A.; Kilbinger, A. F. M. J. Am. Chem. Soc. 2018, 140, 3181.

(28) Zhang, T. Q.; Fu, L. B.; Gutekunst, W. R. Macromolecules 2018, $51,6497$.

(29) Yasir, M.; Liu, P.; Tennie, I. K.; Kilbinger, A. F. M. Nat. Chem. 2019, $11,488$. 